\title{
A Stochastic Surgery Sequencing Model Considering the Moral and Human Virtues
}

\author{
Gerami Farzad $^{1} \&$ Saidi Mehrabad Mohammad ${ }^{2}$ \\ ${ }^{1}$ Department of Industrial Engineering, Iran University of Science and Technology, Narmak, Tehran, Iran \\ ${ }^{2}$ Department of Industrial Engineering, Iran University of Science and Technology, Narmak, Tehran, Iran \\ Correspondence: Gerami Farzad, Department of Industrial Engineering, Iran University of Science and \\ Technology, Narmak, Tehran, Iran. E-mail: gerami@iust.ac.ir/mehrabad@iust.ac.ir
}

Received: December 27, 2015

Accepted: May 8, 2016

Online Published: June 7, 2016

doi:10.5539/mas.v10n9p68

URL: http://dx.doi.org/10.5539/mas.v10n9p68

\begin{abstract}
In this research, we will work sequencing problem of patients demanding surgery under uncertainty in times (including the surgical time, time to prepare the operating room, patient awake time before transferring to the recovery room ...). For this problem, a stochastic mixed integer programming model as Stochastic Surgery Sequencing Model $\left(\mathrm{S}^{3} \mathrm{M}\right)$ has been developed.Since this model is achance-constraint problem, which makes it very complex. This problem aims to minimize the cost of operating room personnel overtime and to reduce patient's waiting time. In mathematical schedule models, we consider three level of patient's priority $\left(F_{p}^{1}, F_{p}^{2}, F_{p}^{3}\right)$. Based on these moral and human dimensions, decision maker can prioritize patients. Restrictions on the balance the operating roomsand priorities for patients are all from the real-world constraints, are included in this issue. A branch-reduce-cut algorithm is used to solve the model.
\end{abstract}

Keywords: sequencing of the patients, stochastic mixed integer programming, chance-constraint problems

\section{Introduction}

A large share of the costs and revenues of a hospital is related to operating rooms. Thus increasing productivity within the operating rooms has a significant impact on the ultimate performance of a hospital. Also improved performance in operating rooms causes increasing economic indicators in hospital, rate of services and also patient's satisfaction. Researchers in planning and scheduling of operating rooms fields have tried to reduce down time of resources, personnel overtime costs, operating room equipment fixed costs, increase revenue and service rates.

Gerami and Saidi-Mehrabad (2014) have brought in their research that surgeries planning and scheduling follows the two steps.The first phase (surgery planning) involves the allocation of elective surgeries to various periods during the planning period, usually weekly, in order to minimize the associated costs. In the second phase (surgery scheduling), sequencing, resource allocation and the surgery start time on a specified date are determined.

In this study, the second phase or the sequencing of elective surgery (inpatient and outpatient) under uncertainty surgery time and other times related surgery will be considered.

Researches related to the Boldy (1976), Blazewich et al. (1983) and Smith et al. (1988) can be a kind of basic research in the areas of planning and scheduling of operating rooms.

Some writers like Magerlein and Martin (1978), have classified literature based on solving methods.

Based on operating room efficiency and patient waiting time, Dexter et al. (2004) reviewed the literature on the operational decisions of management in the operating room. In this research, Decisions were classified into 4 categories and also 15 scenarios were introduced.

But Cardoen et al. (2010) presented more comprehensive classification in six areas related to the characteristics (such as performance measures or types of patients) and technical parameters (e.g., solution methods or considering uncertainties) and they reviewed articles in this field.

In Weiss (1990), Denton et al. (2007), Mancilla \& Store (2009) and Batun et al. (2011) the simultaneous determination of the literature of the sequence and timing of surgery has been studied. 
Lebowitz (2003), Arnaout and Kulbashian (2008), Gul et al. (2011), using techniques such as simulation, are discussed the sequencing of operations under uncertainty in the duration of operation on one or more sources. Chen et al. (2010) write, most of the scheduling in the operating room is done based on experience and innovation. This can cause excessive overtime and poor performance of personnel in the use of the resources.

Liu et al. (2011) used open scheduling strategy. Accordingly, they defined a day (from 9 to 20) in the form of time slots (one-hour). As well they considered the maximum duration of surgery to reduce patient waiting time.They have developed a new heuristic algorithm to solve the problem and achieved the desired results.

Su et al. (2011), based on block scheduling strategy, presented an approach for solving the scheduling problem in operating room and was evaluated using simulation. They call this approach the SOMO (In continuation of previous research $(\mathrm{SOM}))$. In SOMO, the weight vector of a neuron $\left(w_{j}\right)$ indicates a feasible solution to the optimization problem. The best solution is achieved by updating the weight vector (closer to winning neuron weight vectors). This problem is considered for elective patients and also under certainty.

Souki (2011) considered the uncertainty in duration of surgery time and presented two scheduling models for operating room with fuzzy parameters. The first model aims to minimize the completion time of surgery, and the second model aims to minimize the total weighted completion time of patients. He used genetic algorithms and greedy search algorithm to solve these problems.

Ghazalbash and others (2012) presented a model for the daily schedule in operating room under certainty for elective patients in educational hospitals. They have developed a problem with the objective of minimizing the total idle time and solved it by using mixed integer programming.

Dexter et al. (2003), with the aim of increasing the efficiency of the operating room, analyzed different scenarios using event-based simulation. In this model the duration of surgery and also patient arrival was uncertain.

Chaabane et al. (2008) wrote that operating rooms with appropriation more than $10 \%$ of operating budget, are the most expensive part of a hospital. This paper introduces two methods for operating room planning and compares them.

Belien and Demeulemeester (2007) in their research focused on the nurse's working pressure of the operating room due to the workload. They offered a model that integrates the process of scheduling nurses and operating room. The idea of this model is that, firstly, due to surgery constraints, an MSS (Note 1) is produced. Then, taking into account the contribution of different types of surgeries, nursing workload distribution is obtained.

Nunes et al. (2009) using a Markov decision process, were modeled elective patients admission control and using the value iteration algorithm, implemented hypothetical examples.

In some studies, researchers are trying to create an integrated model of planning and scheduling. This means that in addition to the operating room (intraoperative sources), they try to focus on restrictions after surgery rooms. It is closer to the real world. Then attempt to review the literature on planning and scheduling problems in the operating room with consideration of restrictions during and after surgery.

Calichman (2005), Belien and Demeulemeester (2008) and Belien et al. (2009) were able to develop planning and scheduling problems with regard to the limitations and conditions of during and after surgery.

Mulholland et al. (2005) were scheduled surgeries considering the limitations of recovery room, ICU (Note 2) and ward. This study was under uncertainty and researchers have developed a multi criteria model with a financial goal. They have solved these models using real data and linear programming.

Oostrum et al. (2008) believe that an imbalanced schedule in the operating room, leading to fluctuating demand in other departments after the operating room, such as wards and ICU. They were suggesting a MSS (Note 3) in relation to this issue. They were graded requirements for hospital beds in surgical wards and ICU in two phases. In this case, the duration of surgery (for elective patients) was considered uncertain.

Cardoen et al. (2009) presented a multi criteria objective function which minimizes the use of recovery beds and overtime in recovery. Limited access to equipment and additional cleaning of the operating room (e.g., postoperative infection) in this case is considered.To solve the problem of combinatorial optimization, column generation method is applied. On the other hand, a dynamic programming algorithm is presented to solve the problem of bounds.

Shamayleh et al. (2012) considered the issue of operating room capacity planning decisions on the combination of open operating rooms. Researchers in this paper used the integer programming model, in order to combine the 
operating room capacity decisions and operating room time allocation decisions. Capacity decisions include determining combination of the number of operating room that must be opened and the amount of overtime (to meet demand). It was a certain decision model for elective patients.

Saremi et al. (2013) considered a several stages scheduling problem (allocation of surgical services to outpatient). In this model, services time are stochastic.

Gerami and Saidi-Mehrabad (2014) in their study considered the issue of allocation of elective patients (In continuation of their previous work that presented a stochasticmixed integer programming model called SSAM (Note 4)). In this study, they developed a stochastic mixed integer programming model called ISSAM (Note 5). This model includes restrictions on the surgical wards and ICU. Also the duration time of surgery and other times (operating room preparation time, patient preparation time after anesthesia, patient awake time before transferring to the recovery room and operating room cleaning time) are stochastic.

Meskens et al. (2013) presented a multi objective model based on the desires and requirements of the surgical team. Their goals were minimizing the time to do things, minimizing overtime time and maximize the efficacy of the surgical team and solved it by the genetic algorithm in real world. Their strategy was block scheduling strategy. The problem is proposed for elective patients and under certainty.It also assumes all the equipment and materials needed are available.

Based on the above, the present inventions and features of this work can be mentioned the following:

- Considering human dimensions such as:

$\checkmark \quad$ Patient rights prism (the model is intended for patients three levels of priority and pay special attention to the rights of vulnerable groups such as children, pregnant women, the elderly, psychiatric patients, prisoners, the mentally and physically disabled and those without head)

$\checkmark \quad$ The principles of medical ethics

$\checkmark$ Justice

$\checkmark \quad$ Individual freedom (for each scheduling model, a revised approach will be considered, for example after the initial response, if the surgeon had a special view, again, scheduling is done according to the surgeon)

$\checkmark$ Moral

$\checkmark \quad$ Patient and surgeon satisfaction and other human factors satisfaction

In mathematical schedule models, we consider three level of patient's priority $\left(F_{p}^{1}, F_{p}^{2}, F_{p}^{3}\right)$. Based on these moral and human dimensions, decision maker can prioritize patients.

- Stochastic surgery time

- Stochastic other time such as operating room cleaning, operating room preparation time, patient preparation time after anesthesia, patient awake time before transferring to the recovery room

- Separation surgery time of other times (the surgeon is present only during surgery in the operating room)

- Time balancing in the use of operating rooms

- Balancing on allocation of patients with priority to operating rooms

- Considering the preference for special patients such as travelers, children, the elderly and people with disabilities

In this study, we present a problem that sequences the patients demanding surgery under uncertainty, considering real life constraints including priorities, balancing .... In the second part of this research, we present the statement of the problem and develop a mathematical model for our problem. The third section presents the methodology of problem solving. Calculations and numerical results are presented in Section IV. Finally, section V contains the conclusions and recommendations.

\section{Problem Statement}

In this issue, the sequencing of elective patients (inpatient and outpatient) demanding surgery is considered. In other words, based on the list of patients (surgeries list) and taking into account the limitations, for each patient the sequence of surgery will be determined. It tries to increase efficiency of model by considering real-world constraints. Goal and constraints are discussed below. In this section we describe and develop a SMIP (Note 6) model for sequencing of elective surgery. This model is named as $\mathrm{S}^{3} \mathrm{M}$ (Note 7). 


\subsection{Symbols}

The following symbols are used in this paper:

\section{Indices:}

$p$ : Patient (surgery)

$o$ : Operating room

$s$ : Surgeon

\section{Parameters:}

$N$ : The number of patients demanding surgery

$P$ : Number of patients

0 : Number of operating rooms

\section{$S$ : Number of surgeons}

$T_{o}$ : Normal duration of the operating room $o$ (minute)

$T a_{o}$ : Allowed overtime of the operating room $o$ (minute)

$d s_{p}$ : Operating room preparation time of patient $p$ (including operating room preparation time, patient preparation time, anesthesia time)

$f_{d s_{p}}(x)$ : Probability distribution function of $d s_{p}$

$d a_{p}$ : Surgery time of patient $p$

$f_{d a_{p}}(x)$ : Probability distribution function of $d a_{p}$

$d p o_{p}$ : The duration of the process of care after anesthesia for patient $p$ which is done in the operating room

$f_{d p o_{p}}(x)$ : Probability distribution function of $d p o_{p}$

$d c_{p}$ : Operating room cleaning time of patient $p$

$f_{d c_{p}}(x)$ : Probability distribution function of $d c_{p}$

$D_{p}: D_{p}=d s_{p}+d a_{p}+d c_{p}$

$f_{D_{p}}(x)$ : Probability distribution function of $D_{p}$

$\left[L_{s}, U_{s}\right]$ : Presence time of surgeon $s$

OT: Opening time of department of Surgery on day $\mathrm{t}$

$M, M_{1}, M_{2}, M_{3}$ : A Large number

\section{Decision variables:}

$x_{p, o}: 1$ if patient $p$ is allocated to operating room $o, 0$ otherwise

$\xi_{p, p}: 1$ if the patient $p$ is entering the operating room before the patient $p^{\prime}, 0$ otherwise

$\pi_{p, s}: 1$ when surgeon $s$ is allocated to patient $p$ (to perform operation), 0 otherwise

$F_{p}^{1}: 1$ when the patient $p$ has the lowest priority for surgery on day t, 0 otherwise

$F_{p}^{2}: 1$ when the patient $p$ has the medium priority for surgery on day t, 0 otherwise

$F_{p}^{3}: 1$ when the patient $p$ has the highest priority for surgery on day t, 0 otherwise

$\lambda_{p}: 1$ when the patient $p$ is a man, 0 otherwise

\section{Positive real variable:}

$t o_{p}$ : Start time in operating room for patient $p$

\subsection{Sequencing Mathematical Model for Surgical Cases}

The conceptual model that is used in this problem is shown in figure 1. In this figure the entrance time and exit time of the patient and also the entrance time and exit time of the surgeon in a operating room have been shown. 


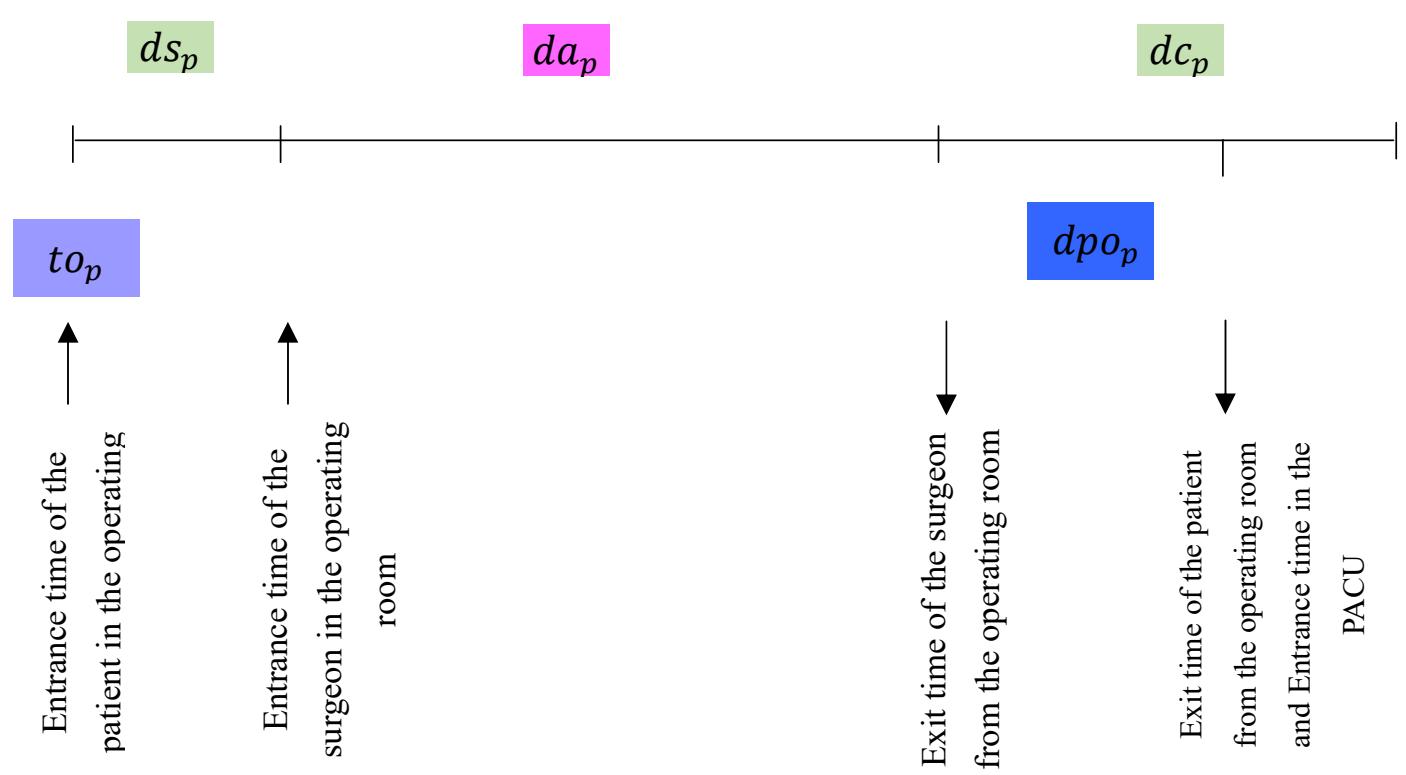

Figure 1. Conceptual model (the entrance and exit of the patient and the surgeon)

This model aims to minimize the sum of start time in operating room (formula (1)). According to patient's priority, $M_{1}, M_{2}$ and $M_{3}$ are different. So this model makes the most effort to minimize the operation start time of patients with a higher priority.

$$
\operatorname{Min} x_{o}=M_{1} \cdot \sum_{p=1}^{N} F_{p}^{1} \cdot t o_{p}+M_{2} \cdot \sum_{p=1}^{N} F_{p}^{2} \cdot t o_{p}+M_{3} \cdot \sum_{p=1}^{N} F_{p}^{3} \cdot t o_{p}
$$

Constraints of this model are formulated as follows:

$$
\begin{aligned}
& \left(\mathrm{to}_{\dot{\mathrm{p}}}+\mathrm{ds} \mathrm{s}_{\dot{\mathrm{p}}}\right)-\left(\mathrm{to}_{\mathrm{p}}+\mathrm{ds} \mathrm{s}_{\mathrm{p}}+\mathrm{da} \mathrm{a}_{\mathrm{p}}\right) \geq \mathrm{M}\left(\pi_{\mathrm{p}, \mathrm{s}}+\pi_{\dot{\mathrm{p}}, \mathrm{s}}+\xi_{\mathrm{p}, \dot{\mathrm{p}}}-3\right) \forall \mathrm{p}, \dot{\mathrm{p}} \in[1, \ldots, \mathrm{N}], \mathrm{p} \neq \dot{\mathrm{p}}, \forall \mathrm{s} \in[1, \ldots, \mathrm{S}] \\
& \text { to }_{\mathrm{p}}+\mathrm{ds}_{\mathrm{p}} \geq \pi_{\mathrm{p}, \mathrm{s}} \cdot \mathrm{L}_{\mathrm{s}} \forall \mathrm{p} \in[1, \ldots, \mathrm{N}], \forall \mathrm{s} \in[1, \ldots, \mathrm{S}] \\
& \text { to }_{\hat{p}}-\left(\text { to }_{p}+D_{p}+\mathrm{dpo}_{\mathrm{p}}\right) \geq \mathrm{M}\left(\mathrm{x}_{\mathrm{p}, \mathrm{o}}+\mathrm{x}_{\hat{\mathrm{p}}, \mathrm{o}}+\xi_{\mathrm{p}, \dot{p}}-3\right) \forall \mathrm{p}, \dot{\mathrm{p}} \in[1, \ldots, \mathrm{N}], \mathrm{p} \neq \dot{\mathrm{p}}, \forall \mathrm{o} \in[1, \ldots, 0] \\
& \xi_{\mathrm{p}, \dot{p}}+\xi_{\mathrm{p}, \mathrm{p}}=1 \forall \mathrm{p}, \dot{\mathrm{p}} \in[1, \ldots, \mathrm{N}], \mathrm{p}>\dot{\mathrm{p}} \\
& \text { to }_{\dot{\mathrm{p}}}-\text { to }_{\mathrm{p}} \geq \mathrm{M}\left(\xi_{\mathrm{p}, \dot{\mathrm{p}}}-1\right) \forall \mathrm{p}, \mathrm{p} \in[1, \ldots, \mathrm{N}], \mathrm{p} \neq \dot{\mathrm{p}} \\
& t o_{\dot{p}}-t o_{p} \geq 0 \forall p, p \in[1, \ldots, \hat{N}], \mathrm{p} \neq \dot{\mathrm{p}}, F_{p}>F_{\dot{p}} \\
& \xi_{p, \dot{p}}=1 \forall p, p \in[1, \ldots, \mathrm{N}], \mathrm{p} \neq \dot{\mathrm{p}}, F_{p}>F_{\dot{p}} \\
& F_{p}^{1}+F_{p}^{2}+F_{p}^{3}=1 \forall p=1,2, \ldots, P \\
& \xi_{p, p}=1 \forall p, p \in[1, \ldots, N], p \neq \dot{p}, F_{p}^{3}=1, F_{p^{\prime}}^{3}=0 \\
& \xi_{p, \dot{p}}=1 \forall p, p \in[1, \ldots, N], p \neq \dot{p}, F_{p}^{2}=1, F_{p^{\prime}}^{1}=1 \\
& \mathrm{dpo}_{\mathrm{p}} \geq \mathrm{o} \forall \mathrm{p} \in[1, \ldots, \mathrm{N}] / \mathrm{tw}_{\mathrm{p}}, \mathrm{to}_{\mathrm{p}}, \mathrm{tp}_{\mathrm{p}} \geq \mathrm{OT} \forall \mathrm{p} \in[1, \ldots, \mathrm{N}] \\
& \mathrm{x}_{\mathrm{p}, \mathrm{o}} \in\{0,1\} \forall \mathrm{o} \in[1, \ldots, 0], \forall \mathrm{p} \in[1, \ldots, \mathrm{N}] \\
& \xi_{\mathrm{p}, \dot{p}} \in\{0,1\} \forall \mathrm{p}, \dot{\mathrm{p}} \in[1, \ldots, \mathrm{N}], \mathrm{p} \neq \dot{\mathrm{p}} \\
& \pi_{p, s} \in\{0,1\} \forall s=1,2, \ldots, S, \forall p=1,2, \ldots, P \\
& \varepsilon_{p, o} \in\{0,1\} \forall o=1,2, \ldots, O, \forall p=1,2, \ldots, P \\
& \lambda_{\mathrm{p}} \in\{0,1\} \forall \mathrm{p} \in[1, \ldots, \mathrm{N}] \\
& F_{p} \in\{0,1\} \forall p=1,2, \ldots, P
\end{aligned}
$$

The constraint (2) ensures that the overlap does not occur for each two patients were allocated to a surgeon. The constraint (3) checks the start time of surgery considering the presence time of surgeon.Formula (4) refers to the lack of overlap between the two cases which have been allocated to an operating room.Constraint (5) and (6) checks the transposition in patient entrance to operating room.Constraints (7) to (11) ensure the patient's priority to entrance to operating room. 


\section{Problem Solving Methodology}

Since this model is a chance-constraint problem, which makes it very complex. Based on the proposed model by Cheon et al. (2006), the following method (that is a branch reduce cut algorithm) is developed to solve the problem.

Accordingly, the problem becomes from A to B.

A)

$$
\begin{gathered}
\min c^{T} x \\
P(\{\xi \mid T x \geq \xi\}) \geq \alpha \\
A x=b \\
x \geq 0
\end{gathered}
$$

B)

$$
\begin{gathered}
\min c^{T} x \\
T x \geq \xi \geq y \\
A x=b \\
\sum_{k=1}^{K} p^{k} \lambda_{k} \geq \alpha \\
\xi_{j}^{k} \lambda_{i} \leq y_{j} j=1, \ldots, m k=1, \ldots, K \\
\lambda_{k} \in\{0,1\} k=1, \ldots, K \\
x \geq 0, y \geq 0
\end{gathered}
$$

In this model, $\xi(\omega)$ has $k$ possible realizations $\left\{\xi_{j}, \ldots, \xi_{k}\right\}$ with probabilities $\left\{p_{1}, \ldots, p_{k}\right\}$. Therefore, using sampling and simulation techniques, we can calculate the probability distribution functions. According to Zhou and Dexter (1998) and May et al. (2000) duration of surgery can be approximated with the log- normal distribution function.

Log-normal distribution is a distribution that its natural logarithm is a normal distribution with $\boldsymbol{\mu}$ and $\boldsymbol{\sigma}$ parameters. Mean and variance of the log-normal distribution function, respectively, are calculated by the following formula:

$$
\begin{gathered}
e^{\mu+\sigma^{2} / 2} \\
\left(e^{\sigma^{2}}-1\right) e^{2 \mu+\sigma^{2}}
\end{gathered}
$$

Based on sampling and simulations techniques, distribution functions for other times are calculated that it is given in Section 4.1. The calculation results are presented in the next sections.

\section{Numerical Experiments}

In this section, using the actual data associated with a first class state hospital, the model is tested and the results are presented.

\subsection{Data}

To evaluate the proposed model, 28 samples have been tested in various sizes.This issue has been considered three operating rooms. Usual opening time and closing time of surgical center is 7 to 14 (420 minutes) and allowed overtime of each operating Room is 180 minutes.In maximal condition, there are 7 surgeons related to 5 surgeries type, including OPT (Note 8), ENT(Note 9), ORT (Note 10), GEN (Note 11), OBG (Note 12). The maximum working time of surgeons is equal to 480 minutes. If we assume that surgical center is opened at time zero, then the operation's timeframe for each surgeon is $[0,480]$. In this hospital, minimum time for surgery in about 30 minutes and the maximum time is about 300 minutes. Information about the some parameters is provided in table 1. Based on sampling and simulations techniques, we can approximate the distribution function of the surgery duration and the other time (preparation time in operating room, cleaning time after each surgery, patient preparation time after anesthesia, patient awake time before transferring to the recovery room,...) to log-normal distribution function. The means and variances of these durations is given in table 2 . Table 3 continues row 10 in table 1 . It is necessary to note that part of the patient preparation time after anesthesiais overlap with the 
preparation of the operating room.

Table 1. The value of some parameters

\begin{tabular}{|c|c|c|c|}
\hline Row & Parameter & Value & Comments \\
\hline 1 & $N$ & 9 to 16 & depending on the scenario \\
\hline 2 & $P$ & $p \in[1, \ldots, N]$ & \\
\hline 3 & 0 & 3 (room) & \\
\hline 4 & $S$ & 7 (person) & \\
\hline 5 & $T_{o}$ & 420 (minute) & \\
\hline 6 & $T a_{o}$ & 180 (minute) & \\
\hline 7 & $L_{s}$ & $7(\mathrm{AM})$ & \\
\hline 8 & $U_{s}$ & $15(\mathrm{PM})$ & \\
\hline 9 & OT & $7(\mathrm{AM})$ & \\
\hline 10 & $\pi_{p, s}$ & 0 or 1 & $\begin{array}{l}\text { As an example in one of the scenarios that } \mathrm{N}=10 \text {, } \\
\pi_{p, s} \text { is given in table } 3 .\end{array}$ \\
\hline 11 & $\lambda_{p}$ & 0 or 1 & $\begin{array}{l}\text { As an example in one of the scenarios that } \mathrm{N}=10 \text {, } \\
\lambda_{p} \text { is considered as follow: } \\
\text { For } \mathrm{P}=1,2,5,8,9 \lambda_{n}=1 \text {, Others } \lambda_{n}=0\end{array}$ \\
\hline 12 & $F_{p}^{3}$ & 0 or 1 & $\begin{array}{l}\text { As an example in one of the scenarios that } \mathrm{N}=10 \text {, } \\
F_{p}^{3} \text { is considered as follow: } \\
\text { For } \mathrm{P}=2,3,8,10 F_{p}^{3}=1 \text {, Others } F_{p}^{3}=0\end{array}$ \\
\hline
\end{tabular}

Table 2. The means and variances of durations (log-normal distribution)

\begin{tabular}{lcll}
\hline Row & Stochastic parameter & Mean & Standard deviation \\
\hline 1 & $d s_{p}$ & 30 & 10 \\
2 & $d a_{p}$ & 90 & 30 \\
3 & $d p o_{p}$ & 15 & 5 \\
4 & $d c_{p}$ & 15 & 5 \\
\hline
\end{tabular}

Table 3. The value of $\pi_{p, s}$ (in one of the scenarios that $\mathrm{N}=10$ )

\begin{tabular}{llllllll}
\hline $\boldsymbol{\pi}_{\boldsymbol{p}, \boldsymbol{s}}$ & $\mathbf{1}$ & $\mathbf{2}$ & $\mathbf{3}$ & $\mathbf{4}$ & $\mathbf{5}$ & $\mathbf{6}$ & $\mathbf{7}$ \\
\hline $\mathbf{1}$ & 1 & 0 & 0 & 0 & 0 & 0 & 1 \\
$\mathbf{2}$ & 0 & 0 & 1 & 0 & 0 & 0 & 0 \\
$\mathbf{3}$ & 0 & 0 & 0 & 1 & 1 & 0 & 0 \\
$\mathbf{4}$ & 0 & 1 & 0 & 0 & 0 & 0 & 0 \\
$\mathbf{5}$ & 1 & 0 & 0 & 0 & 0 & 0 & 1 \\
$\mathbf{6}$ & 0 & 0 & 0 & 1 & 1 & 0 & 0 \\
$\mathbf{7}$ & 0 & 0 & 0 & 1 & 1 & 0 & 0 \\
$\mathbf{8}$ & 1 & 0 & 0 & 0 & 0 & 0 & 1 \\
$\mathbf{9}$ & 0 & 0 & 0 & 0 & 0 & 1 & 0 \\
$\mathbf{1 0}$ & 1 & 0 & 0 & 0 & 0 & 0 & 1 \\
\hline
\end{tabular}

\subsection{Results}

The models were coded in MATLAB 2014a and solved with a heuristic algorithm. We run programs on an Intel $\mathbb{R}$ core $^{\mathrm{TM}}$ i7-4500U CPU @ $1.80 \mathrm{GHz} 2.40 \mathrm{GHz}$ processor and 8 GB RAM.The results are presented in Table 4. To evaluate the proposed model $\left(\mathrm{S}^{3} \mathrm{M}\right.$ (Note 13)), 28 samples were considered, that the number of patients on the waiting list was between 7 to 15 and the number of surgeons was from 4 to 7 . However, the objective function value for each of the scenarios is estimated $\left(M_{1}=100000, M_{2}=500000, M_{3}=1000000\right)$. 
Table 4. Results of 28 samples

\begin{tabular}{cccc}
\hline Sample & Patients & Surgeons & Value of goal function (rounded) (minutes) \\
\hline 1 & 15 & 7 & 583800000 \\
2 & 13 & 6 & 481400000 \\
3 & 7 & 6 & 236000000 \\
4 & 8 & 6 & 308000000 \\
5 & 10 & 4 & 401300000 \\
6 & 7 & 5 & 265600000 \\
7 & 9 & 6 & 362100000 \\
8 & 8 & 7 & 302000000 \\
9 & 10 & 4 & 395100000 \\
10 & 12 & 5 & 438600000 \\
11 & 7 & 5 & 259700000 \\
12 & 14 & 7 & 546300000 \\
13 & 8 & 6 & 297700000 \\
14 & 15 & 5 & 606200000 \\
15 & 9 & 5 & 333400000 \\
16 & 14 & 7 & 559200000 \\
17 & 12 & 7 & 418100000 \\
18 & 11 & 5 & 393800000 \\
19 & 9 & 6 & 376300000 \\
20 & 10 & 4 & 392700000 \\
21 & 7 & 4 & 236200000 \\
22 & 11 & 7 & 372100000 \\
23 & 9 & 6 & 365300000 \\
24 & 13 & 6 & 468400000 \\
25 & 11 & 5 & 396500000 \\
26 & 8 & 7 & 303500000 \\
27 & 13 & 5 & 458600000 \\
28 & 9 & 4 & 334700000 \\
\hline
\end{tabular}

For example, table 5 provides sequence of patients for 3 samples (for reasons of brevity, only 3 examples are presented). On the table in front of each sample, have been presented the sequence of patients for each operating room.

Table 5. Patients sequence after reactive scheduling for 3 samples

\begin{tabular}{|c|c|c|c|c|}
\hline Sample & Number of patients & Operating room & Sequence of patients & processing time (s) \\
\hline 1 & 11 & $\begin{array}{l}o_{1} \\
o_{2} \\
o_{3} \\
\end{array}$ & $\begin{array}{c}\left\{p_{8}, p_{4}, p_{6}\right\} \\
\left\{p_{9}, p_{11}, p_{5}, p_{2}\right\} \\
\left\{p_{10}, p_{1}, p_{7}, p_{3}\right\}\end{array}$ & 228 \\
\hline 2 & 9 & $\begin{array}{l}o_{1} \\
o_{2} \\
o_{3} \\
\end{array}$ & $\begin{array}{l}\left\{p_{2}, p_{6}, p_{8}\right\} \\
\left\{p_{7}, p_{3}, p_{1}\right\} \\
\left\{p_{4}, p_{9}, p_{5}\right\}\end{array}$ & 176 \\
\hline 3 & 14 & $\begin{array}{l}o_{1} \\
o_{2} \\
o_{3} \\
\end{array}$ & $\begin{array}{c}\left\{p_{11}, p_{2}, p_{10}, p_{3}, p_{7}\right\} \\
\left\{p_{1}, p_{5}, p_{14}, p_{4}\right\} \\
\left\{p_{13}, p_{6}, p_{9}, p_{12}, p_{8}\right\}\end{array}$ & 263 \\
\hline
\end{tabular}

For example, Figure 2 is drawn for $o_{3}$ in sample 1 . In the figure 2 , the times are rounded. 


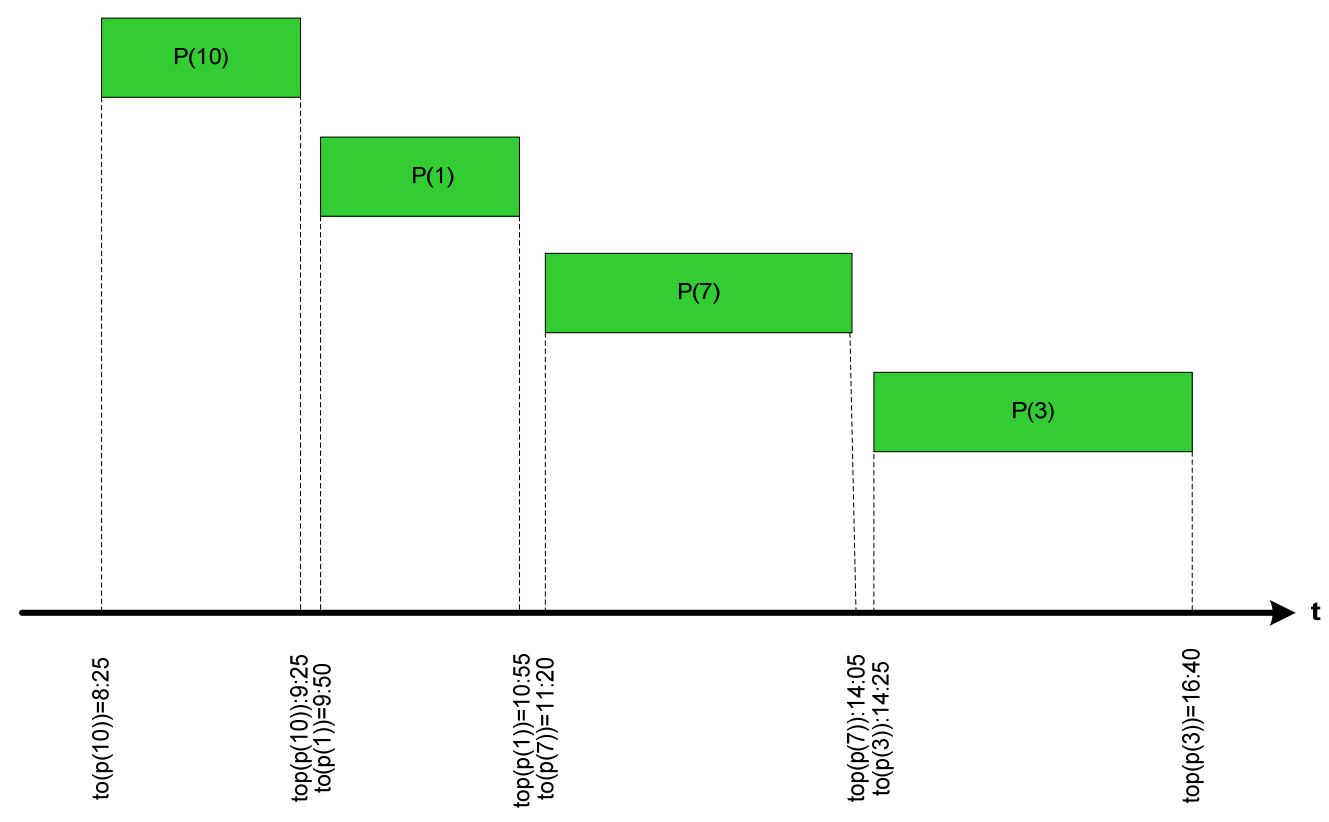

Figure 2. Sequence of patients in operating room $o_{3}$ in sample 1

In Table 6, the results of 15 samples are compared to two methods (current and proposed method).In this table the total waiting time for patients and cancellation (due to long waiting time) have been shown.Each of these models sampled separately.The sampling is per number of patient (between 8 to 12) which each is repeated three times.

Table 6. Evaluations of $\mathrm{S}^{3} \mathrm{M}$ results compared to the current model

\begin{tabular}{llllll}
\hline & & Existent Method & & Proposed Method $\left(\mathbf{S}^{\mathbf{3}} \mathbf{M}\right)$ & \\
\cline { 3 - 6 } Sample & Patients & Total waiting time (minutes) & Cancelation & Total waiting time (minutes) & Cancelation \\
\hline 1 & 8 & 940 & 0 & 190 & 0 \\
2 & 8 & 775 & 0 & 270 & 0 \\
3 & 8 & 815 & 0 & 235 & 0 \\
4 & 9 & 1115 & 0 & 360 & 0 \\
5 & 9 & 985 & 0 & 325 & 0 \\
6 & 9 & 1010 & 0 & 365 & 0 \\
7 & 10 & 1760 & 1 & 445 & 0 \\
8 & 10 & 1570 & 0 & 515 & 0 \\
9 & 10 & 1905 & 0 & 570 & 0 \\
10 & 11 & 2310 & 1 & 525 & 0 \\
11 & 11 & 2220 & 0 & 520 & 0 \\
12 & 11 & 1985 & 0 & 525 & 0 \\
13 & 12 & 2280 & 0 & 635 & 0 \\
14 & 12 & 2215 & 1 & 570 & 0 \\
15 & 12 & 2355 & 0 & 665 & 0 \\
\hline
\end{tabular}

Comparing the results listed in Table 6 has been performed by diagram 1 and2. In diagram 1, the total waiting time of the current method and $\mathrm{S}^{3} \mathrm{M}$ (Note 14) is presented. For every 15 cases sampled, this chart indicates that the proposed model is much lower waiting time than existing method.It reflects the higher efficiency of the proposed model. 


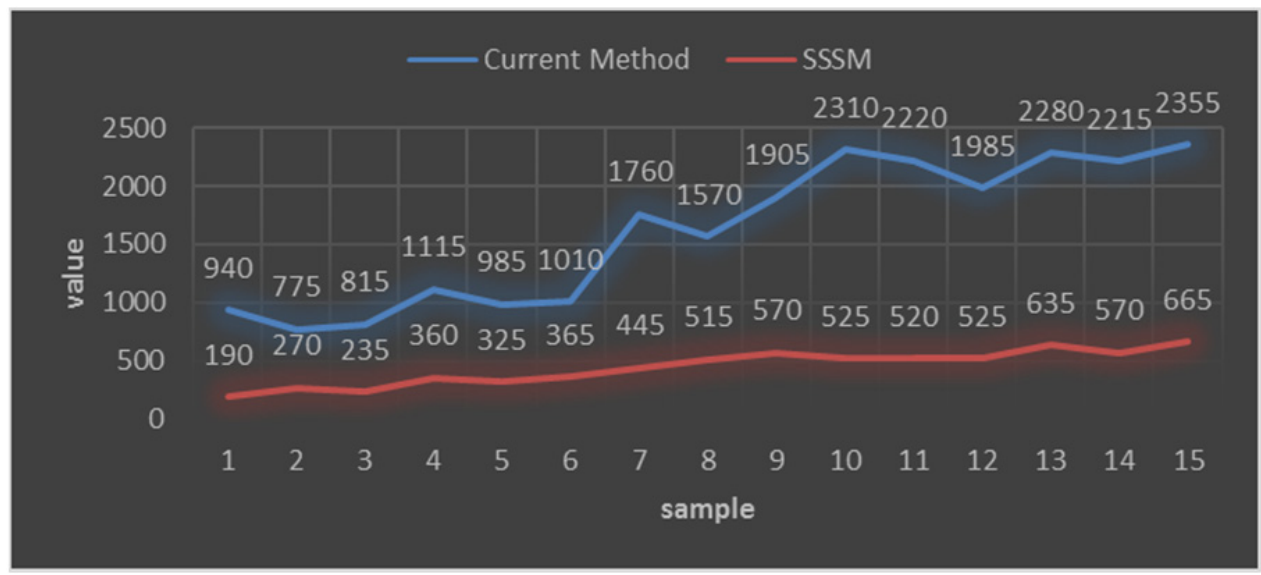

Diagram 1. Comparison of total waiting time of the current method and $\mathrm{S}^{3} \mathrm{M}$

In diagram 2, the number of cancelation (due to long waiting time) of the current method and $\mathrm{S}^{3} \mathrm{M}$ is presented.The proposed model shows less cancelation than the existing model.

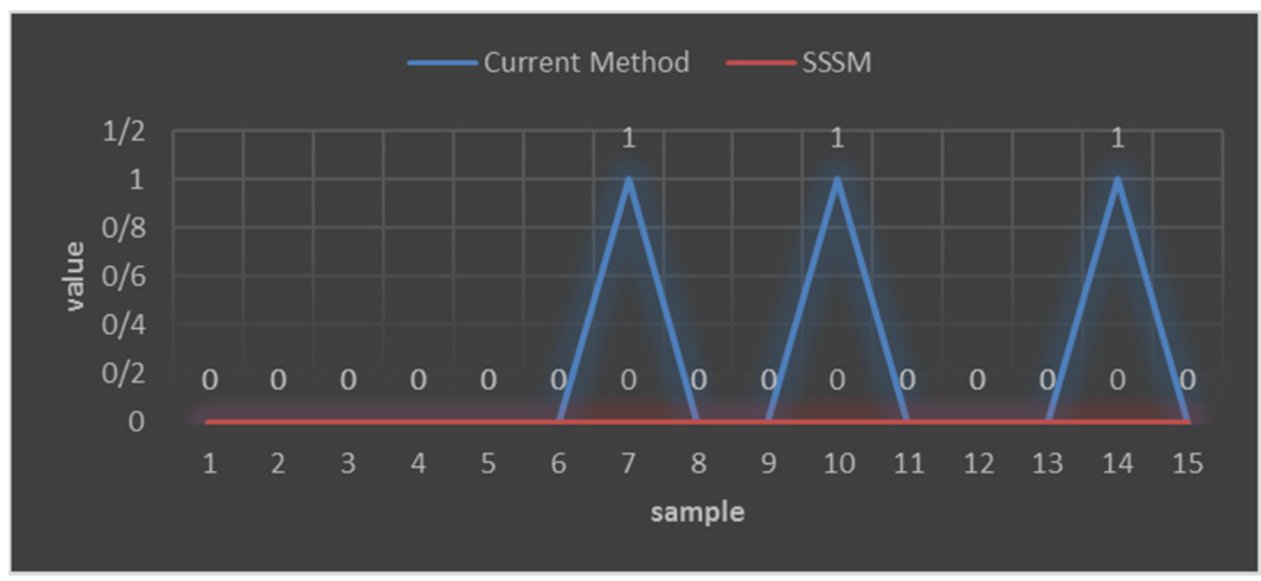

Diagram 2. Comparison of cancelation (due to long waiting) of the current method and $\mathrm{S}^{3} \mathrm{M}$

Also in Table 7, the average of total waiting time, average of cancelation, average of free capacity in waiting room (men/women) and the average of free capacity in PACU of the current method and $\mathrm{S}^{3} \mathrm{M}$ are presented.

Table 7. Average results of the current method and $S^{3} M$

\begin{tabular}{lll}
\hline & Existent Method & Proposed Method $\left(\mathbf{S}^{\mathbf{3}} \mathbf{M}\right)$ \\
\hline Average of total waiting time & 1616 & 447.67 \\
Average of cancelation & 0.20 & 0 \\
\hline
\end{tabular}

As can be seen, the average of total waiting time in the $S^{3} \mathrm{M}$ shows a decline about 72 percent and also results show a decrease from 0.11 to 0 in the average of cancelation.

\section{Conclusions}

In this paper, a stochastic mixed integer programming mathematical model presented for the sequencing of patients in operating rooms. This article has a special approach in some aspects, such as the human dimension, justice, virtue, ethics, individual freedom and rights of vulnerable groups of society, including children, pregnant women, and elderly. In mathematical schedule models, we consider three level of patient's priority $\left(F_{p}^{1}, F_{p}^{2}, F_{p}^{3}\right)$. Based on these moral and human dimensions, decision maker can prioritize patients. In this model, surgery time, 
operating room preparation time, patient preparation time after anesthesia, patient awake time before transferring to the recovery room and operating room cleaning time is stochastic. Some restrictions such as balance the operating roomsand priorities for patients that are all from the real-world constraints, are included in this issue. In order to solve the model, a branch reduce cut algorithm was used. The proposed model ( $\mathrm{S}^{3} \mathrm{M}($ Note 15$\left.)\right)$ was evaluated and verified by solving several real examples of a first class state hospital (see table 4, 5). By comparing the obtained results with real data, we can reach a satisfactory model and thus its excellent performance (see table 6,7 ). For future research, it is suggested that a model is developed considering the non-elective patients (urgent and emergency). It is also suggested that the issue to be reviewed taking into account the limitations of recovery and waiting room. Furthermore, researchers can test the model in other situations such as educational hospitals or private hospitals and examine the performance of the model.

\section{Acknowledgments}

We would like to express our sincere appreciation for the following people: Mrs. Masoumeh Abedini, Mrs. Nasrin Poursakhi, Ali Aboutalebi, doctors and nurses of operating rooms personnel for their valuable help for providing us data for this study.

\section{References}

Arnaout, J. P. M., \& Kulbashian, S. (2008). Maximizing the utilization of operating rooms with stochastic times using simulation, In S. J. Mason, R. R. H. L. Mönch, O. Rose, T. Jefferson, \& J. W. Fowler (Eds.). Proceedings of the 2008 Winter Simulation Conference.

Batun, S., Denton, B. T., Huschka, T. R., \& Schaefer, A. J. (2011). Operating Room Pooling and Parallel Surgery Processing Under Uncertainty. Informs Journal on Computing, 23(2), 220-237.

Belien, J., \& Demeulemeester, E. (2007). Building cyclic master surgery schedules with leveled resulting bed occupancy. European Journal of Operational Research, 176(2), 1185-1204.

Belien, J., \& Demeulemeester, E. (2008). A branch-and-price approach for integrating nurse and surgery scheduling. European Journal of Operational Research, 189(3), 652-668.

Belien, J., Demeulemeester, E. \& Cardoen, B. (2009). A decision support system for cyclic master surgery scheduling with multiple objectives. Journal of Scheduling, 12(2), 147-161.

Blazewich, J., Lenstra, J. K., \& Rinnooy K. A. (1983). Scheduling subject to resource constraints: Classification and complexity. Discrete Applied Mathematics, 5, 11-24.

Boldy, D. (1976). A review of the application of mathematical programming to tactical and strategic health and social services problems. Operational Research Quarterly, 27(2), 439-448.

Calichman, M. V. (2005). Creating an optimal operating room schedule. AORN Journal, 81, 580-588.

Cardoen, B., Demeulemeester, E., \& Belien, J. (2009). Sequencing surgical cases in a daycare environment: An exact branch-and-price approach. Computers and Operations Research, 36(9), 2660-2669.

Cardoen, B., Demeulemeester, E., \& Belien, J. (2010). Operating room planning and scheduling: A literature review. European Journal of Operational Research, 201, 921-932.

Chaabane, S., Meskens, N., Guinet, A., \& Laurent, M. (2008). Comparison of two methods of operating theatre planning: Application in Belgian hospitals. International Conference on Service Systems and Service Management. Troyes.

Chen, C. K., Lin C., Hou T. H., Wang S. H., \& Lin H. M. (2010). A Study of Operating Room Scheduling That Integrates Multiple Quantitative and Qualitative Objectives. Journal of Nursing Research, 18(1).

Cheon, M. S., Ahmed, S., \& Al-khayyal, F. (2006). A branch-reduce-cut algorithm for the global optimization of probabilistically constrained linear programs. Mathematical Programming, 108, 617-634.

Denton, B., Viapiano, J. \& Vogl, A. (2007). Optimization of surgery sequencing and scheduling decisions under uncertainty. Health Care Management Science, 10, 13-24.

Dexter, F., Epstein, R. H., Traub, R. D. \& Xiao, Y. (2004). Making Management Decisions on the Day of Surgery Based on Operating Room Efficiency and Patient Waiting Times. Anesthesiology, 101, 1444-1453.

Dexter, F., Macario, A., Traub, R. D. \& Lubarsky, D. A. (2003). Operating room utilization alone is not an accurate metric for the allocation of operating room block time to individual surgeons with low caseloads. Anesthesiology, 98, 1243-1249.

Gerami, F., \& Saidi-mehrabad, M. (2014). Allocation of patients to operating rooms under uncertainty considering 
the constraints after the operating room. International Journal of Current Life Science, 4, 11869-11876.

Gerami, F., Saidi-mehrabad, M. (2014). Allocation of patients to operating rooms under uncertainty. International Journal of Current Life Science, 4, 7121-7127.

Ghazalbash, S., Sepehri, M. M., Shadpour, P., \& Atighehchian, A. (2012). Operating Room Scheduling in Teaching Hospitals. Advances in Operations Research 2012.

Gul, S., Denton, B. T., Fowler, J. W. \& Huschka, T. (2011). Bi-Criteria Scheduling of Surgical Services for an Outpatient Procedure Center. Production and Operations Management, 20, 406-417.

Lebowitz, P. (2003). Schedule the short procedure first to improve OR efficiency. AORN Journal, 78, 651-654, 657-659.

Liu, Y., Chu, C., \& Wang, K. (2011). A new heuristic algorithm for the operating room scheduling problem. Computers \& Industrial Engineering, 61, 865-871.

Magerlein, J., \& Martin, J. (1978). Surgical demand scheduling: A review. Health Services Research, 13, 418-433.

Mancilla, C., \& Storer, R. H. (2009). Stochastic Sequencing and Scheduling of an Operating Room. MOPTA. Bethlehem, PA.

May, J. H., Strum, D. P., \& Vargas, L. G. (2000). Fitting the Lognormal distribution to surgical procedure times. Decision Sciences, 31, 129-148.

Meskens, N., Duvivier, D., \& Hanset, A. (2013). Multi-objective operating room scheduling considering desiderata of the surgical team. Decision Support Systems.

Mulholland, W., Abrahamse, P., \& Bahl, V. (2005). Linear programming to optimize performance in a department of surgery. Journal of the American College of Surgeons, 200(6), 861-868.

Nunes, L. G. N., De Carvalho, S. V., \& Rodrigues, R. C. M. (2009). Markov decision process applied to the control of hospital elective admissions. Artificial Intelligence in Medicine, 47, 159-171.

Oostrum, J. M., Houdenhoven, M., Hurink, J. L., Hans, E. W., Wullink, G., \& Kazemier, G. (2008). A master surgical scheduling approach for cyclic scheduling in operating room departments. OR Spectrum, 30, 355-374.

Saremi, A., Jula, P., Elmekkawy, T., \& Wang, G. (2013). Appointment scheduling of outpatient surgical services in a multistage operating room department. Int. J. Production Economics, 141, 646-658.

Shamayleh, A., Fowler, G., \& Zhang, M. (2012). Operating Room Capacity Planning Decisions. World Academy of Science, Engineering and Technology, 64, 665-669.

Smith, J. C., Schaefer, A. J., \& Yen, J. W. (1988). A stochastic integer programming approach to solving a synchronous optical network ring design problem. Networks, 44.

Souki, M. (2011). Operating theatre scheduling with fuzzy durations. Journal of Applied Operational Research, 3(3), 177-191.

Su, M. C., Lai, S. C., Wang P. C., Hsieh, Y. Z., \& Lin, H. C. (2011). A SOMO-based approach to the operating room scheduling problem. Expert Systems with Applications, 38, 15447-15454.

Weiss, E. N. (1990). Models for Determining Estimated Start Times and Case Orderings In Hospital Operating Rooms. IIE Transactions, 22, 143-150.

Zhou, J., \& Dexter, F. (1998). Method to assist in the scheduling of add-on surgical cases: Upper prediction bounds for surgical case durations based on the log-normal distribution. Anesthesiology, 89 .

\section{Notes}

Note 1. Master Surgery Schedule.

Note 2. Intensive Care Unit.

Note 3. Master Surgical Schedule.

Note 4. Tochastic Surgery Allocation Model.

Note 5. Integrated Stochastic Surgery Allocation Model.

Note 6. Stochastic Mixed Integer Programming. 
Note 7. Stochastic Surgery sequencing Model.

Note 8. Ophthalmic.

Note 9. Ear, Nose, Throat.

Note 10. Orthopedic.

Note 11. General.

Note 12. Obstetrics \& Gynecology.

Note 13. Stochastic Surgery SequencingModel.

Note 14. Stochastic Surgery Sequencing Model.

Note 15. Stochastic Surgery Sequencing Model.

\section{Copyrights}

Copyright for this article is retained by the author(s), with first publication rights granted to the journal.

This is an open-access article distributed under the terms and conditions of the Creative Commons Attribution license (http://creativecommons.org/licenses/by/3.0/). 